\title{
Vorwort zur 2., verbesserten Auflage
}

In der zweiten Auflage sind Druckfehler korrigiert und einige Aktualisierungen vorgenommen worden. Textbestand und Seitenumbruch sind unverändert. Für Korrekturhinweise danke ich Frederike Eggs und Patricia Sdzuy.

Das Ziel des Readers bleibt die Hinführung zu klassischen Texten der Sprachwissenschaft und zugleich zur neueren Fachliteratur. Er soll zeigen, welche Fragen die Disziplin zusammenhalten und wo Lösungen zu suchen sind. Interessant wird es erst, wenn das Selbstverständlichste - die Alltagssprache - zum Problem geworden ist. Wenn man die Vielfalt der Formen sieht, in denen die Sprachen Wissen übermitteln. Wenn man zeigen kann, warum und wozu jemand etwas so gesagt hat, wie er/sie es gesagt hat. Wer dies erfahren hat, wird sich der Faszination dieser Disziplin kaum entziehen können.

Der Reader ist sehr gut aufgenommen worden. Er hat sich in einführenden Lehrveranstaltungen wie im Selbststudium zur Vorbereitung auf Zwischen- und Abschlußprüfungen bewährt. In - idealerweise vierstündigen oder zweisemestrigen - Grundkursen können einige der grundlegenden und der erweiternden Texte gemeinsam erarbeitet und diskutiert werden. Alle lesen den Text, und eine studentische Kleingruppe stellt ihn kurz vor (etwa 10 Minuten). Kleingruppe und Dozent(in) bereiten die Präsentation in einem Gespräch vor, wobei Fragen zum Text, seine zentralen Gedanken und die Form einer anregenden Präsentation erörtert werden. Die Textpräsentation ist eingebaut in Überblicke (Dozent(in)) und Übungen zur Phonem-, Morphem-, Satz- und Diskursanalyse. Zusätzlich können von den Studierenden eigene Aufnahmen (Wegauskunft, Talkshow, Arbeitsgruppengespräch u.a.) angefertigt, im Ausschnitt verschriftet und ausgewertet werden. Wünschenswert ist ein Tutorium zur Nacharbeit und Vertiefung.

In einem solchen Kurs erscheint Linguistik nicht als Katalog von Termini und Definitionen, die abgefragt und wieder vergessen werden können, sondern als konzentrierte Auseinandersetzung mit einer Sache, die jede(n) angeht. Hier haben eigenständiges Lesen und Erarbeiten wie zündende mündliche Präsentation und argumentative Auseinandersetzung ihren Ort.

Praktisch erprobt sind auch andere Formen: der Lektürekurs mit einer thematischen Gruppe von Texten, die Vorlesung zur neueren Sprachwissenschaft mit dem Reader als Vorlage oder die Arbeit mit einem Einführungsbuch, die durch Texte aus dem Reader ergänzt wird. 